CERN-TH/96-156

UTHEP-96-0602

\title{
Missing Third-Order Leading-Log Corrections in the Small-Angle Bhabha Calculation
}

\author{
S. Jadach \\ Institute of Nuclear Physics, ul. Kawiory 26a, Kraków, Poland \\ CERN, Theory Division, CH-1211 Geneva 23, Switzerland, \\ and \\ B.F.L. Ward ${ }^{\dagger}$ \\ Department of Physics and Astronomy, \\ The University of Tennessee, Knoxville, Tennessee 37996-1200 \\ and SLAC, Stanford University, Stanford, California 94309
}

\begin{abstract}
We calculate the missing third-order leading-log (LL) contribution in the secondorder cross section of the small-angle Bhabha process for a realistic event selection. We find that, for the second-order calculation with Yennie-Frautschi-Suura exponentiaton, the missing third-order LL correction is below $2 \times 10^{-4}$ and is therefore negligible with respect to the current experimental LEP precision, while for the second-order calculation without exponentiaton it can be closer to or even comparable with the experimental precision, at least for certain event selections. The calculation is implemented the LUMLOG Monte Carlo event generator.
\end{abstract}

Submitted to Physics Letters

$\S$ Work supported in part by the US DoE contract DE-FG05-91ER40627, Polish Government grants KBN 2P30225206, 2P03B17210, the European Union under contract No. ERB-CIPD-CT94-0016, and Polish-French Collaboration within IN2P3.

CERN-TH/96-156

UTHEP-96-0602

June 1996 
Total cross section is one of the principal quantities measured at the $\mathrm{Z}$ resonance at LEP1 and also at LEP2. Precision measurement of the total cross section requires an equally precise measurement of the luminosity. The luminosity at LEP is determined experimentally using the Small Angle Bhabha ( $\mathrm{SABH}$ ) process, which is dominated by the t-channel gamma exchange. The best measurements of luminosity have an experimental error [1] of about $8 \times 10^{-4}$. The SABH cross section is, in principle, calculable with an arbitrary precision from Quantum Electrodynamics (QED). The practical evaluation of the $\mathrm{SABH}$ total cross section is quite nontrivial because at this precision level one has to calculate QED radiative corrections up to second or, as we shall see in this work, up to third perturbative order. Since experimental Event Selection (ES) is quite complicated the calculation has to be provided in form of the Monte Carlo (MC) Event Generator (MCEG). In particular, typical luminometer is not capable to see any difference between electrons and photons - that is the corresponding ES is of the calorimetric type.

The subject of calculation of QED radiative corrections is already discussed in many papers, see references in the recent review article in the proceedings of the LEP2 workshop [2]. In this and a shorter summary paper [3], the third-order LL correction, $\mathcal{O}\left(\gamma^{3}\right)$, where $\gamma=2 \frac{\alpha}{\pi}\left[\ln \left(|t| / m_{e}^{2}\right)-1\right]$, is one of important contributions which has to be estimated or better calculated. Potentially, it is as important as the Next-to-Leading-Logarithmic (NLL) second-order contribution, $\mathcal{O}(\alpha \gamma)$. In this paper we shall limit ourselves to pure photonic corrections. We do not discuss pair production at $\mathcal{O}\left(\gamma^{3}\right)$.

The missing $\mathcal{O}\left(\gamma^{3}\right)$ were first quantitatively estimated in [4] as being compatible with zero to within $0.01 \%$. The above was confirmed in [5] and repeated in Ref. [6].

The evaluation of the missing $\mathcal{O}\left(\gamma^{3}\right)$ presented here bears resemblance to an older evaluation of the missing $\mathcal{O}\left(\gamma^{2}\right)$ presented in Ref. [7] and later in Ref. [4]. Let us recall briefly what was done in these works. In Ref. [7] the difference $\mathcal{O}\left(\gamma^{2}\right)-\mathcal{O}\left(\gamma^{1}\right)$, as calculated using the LL structure functions of an electron, was used to evaluate the missing $\mathcal{O}\left(\gamma^{2}\right)$ in the ordinary complete $\mathcal{O}\left(\alpha^{1}\right)$ of refs. $[8,9]^{1}$. The authors of Ref. [4] went one step further - they used the same difference, i.e. $\mathcal{O}\left(\gamma^{2}\right)-\mathcal{O}\left(\gamma^{1}\right)$, aiming to correct $\mathcal{O}\left(\alpha^{1}\right)$ of Ref. [8]. The corrected $\mathcal{O}\left(\alpha^{1}\right)$ cross section of Ref. [8], referred to as OLDBIS + LUMLOG $_{H . O}$., was used for comparisons with the cross section of BHLUMI 2.x Monte Carlo event generator of Ref. [12], which had an $\mathcal{O}\left(\alpha^{1}\right)_{\exp }$ matrix element. More precisely, BHLUMI 2.x was used by all LEP experiments to calculate the SABH cross section while OLDBIS + LUMLOG $_{H . O}$. Monte Carlo ${ }^{2}$ was used in a special test run to evaluate the missing $\mathcal{O}\left(\gamma^{2}\right)$ in BHLUMI 2.x.

In order to better understand what we are doing in the present paper, let us consider a scenario similar to Refs. [4,7] but one order higher. Suppose that, (i) similarly to Ref. [7], we evaluate $\mathcal{O}\left(\gamma^{3}\right)-\mathcal{O}\left(\gamma^{2}\right)$ as an estimate of the missing $\mathcal{O}\left(\gamma^{3}\right)$ in the $\mathcal{O}\left(\alpha^{2}\right) \mathrm{BH}$ -

\footnotetext{
${ }^{1}$ In fact the inspected quantity was $\mathcal{O}\left(\gamma^{\infty}\right)-\mathcal{O}\left(\gamma^{1}\right)$, where $\mathcal{O}\left(\gamma^{\infty}\right)$ was in practice an $\mathcal{O}\left(\gamma^{3}\right)_{\text {exp }}$ parametrization of the infinite order result according to Refs. [10,11]. In any case this difference is dominated by $\mathcal{O}\left(\gamma^{2}\right)$.

${ }^{2}$ The OLDBIS + LUMLOG ${ }_{H . o}$. Monte Carlo of Ref. [4] provides the cross section for arbitrary calorimetric ES. It requires, however, that the cross section is added from two separate MC runs, so it is not an ordinary MCEG.
} 
LUMI calculation and (ii) similarly to Ref. [4] we construct the BHLUMI + LUMLOG H.o. $_{\text {. }}$ cross section as a cross-check of missing $\mathcal{O}\left(\gamma^{3}\right)$ in BHLUMI 4.x with the $\mathcal{O}\left(\alpha^{2}\right)_{\text {exp }}$ matrix element.

First of all we have to remember that contrary to the situation in Ref. [7] where the reference cross section was from the complete $\mathcal{O}\left(\alpha^{1}\right)$ calculation, the present version $4 . x$ of BHLUMI is based on the incomplete $\mathcal{O}\left(\alpha^{2}\right)$ matrix element (no exponentiation!). It has the complete LL $\mathcal{O}\left(\gamma^{2}\right)$ matrix element (of pure photonic type) with incomplete subleading $\mathcal{O}(\alpha \gamma)$ and incomplete sub-sub-leading pure $\mathcal{O}\left(\alpha^{2}\right)$ contributions. This is not, however, any serious obstacle. We could go ahead and realize scenario (i) calculating LUMLOG ${ }_{\text {H.O. being }} \mathcal{O}\left(\gamma^{3}\right)-\mathcal{O}\left(\gamma^{2}\right)$ using the present version of $\mathrm{LUMLOG}^{3}$; this kind of correction will be shown at the end of the present paper. The result is however not so very interesting because in the present version 4.x of BHLUMI we rely mainly on the exponentiated $\mathcal{O}\left(\alpha^{2}\right)_{\text {exp }}$ matrix element, in which we expect most of $\mathcal{O}\left(\gamma^{3}\right)$ contribu-

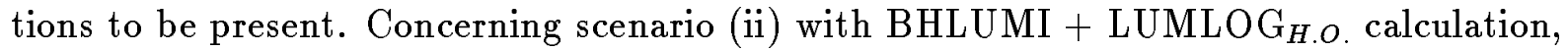
it is also not so attractive as OLDBIS $+\operatorname{LUMLOG}_{H . o}$. because $\mathcal{O}\left(\alpha^{1}\right)$ OLDBIS is an independently written $\mathrm{MC}$ and therefore the comparisons of BHLUMI with OLDBIS + LUMLOG $_{H . O}$. provides a good handle on the technical precision of BHLUMI at the $\mathcal{O}\left(\alpha^{1}\right)$ level. In the comparison of exponentiated BHLUMI versus non-exponentiated BHLUMI $+\mathrm{LUMLOG}_{\text {H.o. }}$. we lose this advantage because the basic MC phase-space integration method for BHLUMI remains the same in both.

From all the above considerations we conclude the following: what is really interesting and feasible is to calculate LUMLOG H.O. $=\mathcal{O}\left(\gamma^{3}\right)_{\text {exp }}-\mathcal{O}\left(\gamma^{2}\right)_{\text {exp }}$ using LUMLOG, which corresponds exactly to a missing $\mathcal{O}\left(\gamma^{3}\right)_{\text {exp }}$ in the multi-photon BHLUMI 4.x [13]. The $\mathcal{O}\left(\gamma^{3}\right)_{\text {exp }}$ part in the above difference is relatively easy and the relevant matrix element has been present in LUMLOG for a long time. The $\mathcal{O}\left(\gamma^{2}\right)_{\text {exp }}$ part of the above difference is non-trivial because, contrary to the case with $\mathcal{O}\left(\gamma^{1}\right)$ in Ref. [7] or $\mathcal{O}\left(\gamma^{2}\right)$ (no exponentiation) in our hypothetic scenario (i), where the corresponding LL structure functions (no exponentiation!) are unique and well known, here we have to know $\mathcal{O}\left(\gamma^{2}\right)_{\text {exp }}$ and implement in LUMLOG the LL "structure functions" of $\mathcal{O}\left(\gamma^{2}\right)_{\text {exp }}$; these have in addition to the complete $\mathcal{O}\left(\gamma^{2}\right)_{\text {exp }}$, exactly the same incomplete $\mathcal{O}\left(\gamma^{3}\right)$ terms as in BHLUMI 4.x of Ref. [13]. There are two immediate questions to be answered: Is it possible? And is it worthwhile? It is possible but non-trivial because it requires to integrate the multi-photon matrix $\mathcal{O}\left(\alpha^{2}\right)_{\exp }$ of BHLUMI over the phase-space not numerically but analytically, keeping track terms up to ${ }^{4} \mathcal{O}\left(\gamma^{3}\right)$. A calculation of this kind was done - details will be presented elsewhere, see Ref. [14] - and here we shall use the relevant results. We have also asked "is it worthwhile?", keeping in mind that an alternative would be just to add the missing $\mathcal{O}\left(\gamma^{3}\right)$ terms in the BHLUMI matrix element. We answer: Yes, it is worthwhile because, firstly, we would need an analytical calculation to check the correctness of the above hypothetic implementation anyway and, secondly, we expect the missing $\mathcal{O}\left(\gamma^{3}\right)$

\footnotetext{
${ }^{3}$ The matrix element in LUMLOG 2.x of Ref. [12] did not feature $\mathcal{O}\left(\gamma^{2}\right)$ without exponentiation. This option is now available in LUMLOG 4.x.

${ }^{4}$ Next-to-leading $\mathcal{O}(\alpha \gamma)$ terms are also kept in the calculation of Ref. [14], but here they are set to zero, after analytical integration over the transverse photon momenta.
} 
to be small - which we can prove; then we can avoid adding unnecessary complication to the BHLUMI matrix element. For the purpose of the rest of the paper let us denote with $\mathcal{O}\left(\gamma^{2}\right)_{B H L}$ the LL calculation in which exactly the same incomplete $\mathcal{O}\left(\gamma^{3}\right)$ terms as in the $\mathcal{O}\left(\gamma^{2}\right)_{\text {exp }}$ BHLUMI 4.x of Ref. [13] are present.

In the following we shall define the content of the $\mathcal{O}\left(\gamma^{2}\right)_{B H L}$ matrix element in the new LUMLOG 4.x (part of BHLUMI 4.x). In order to gradually introduce the notation, let us recall the implementation of the complete $\mathcal{O}\left(\gamma^{3}\right)$ in LUMLOG. Next, we shall define new $\mathcal{O}\left(\gamma^{2}\right)_{B H L}$ differential distributions calculated on Ref. [14]. Let us start with the master formula for the small-angle Bhabha total cross-section where, in addition to the initialstate bremsstrahlung, we have also the final-state bremsstrahlung, both in the $\mathcal{O}\left(\alpha^{r}\right) \mathrm{LL}$ approximation. It reads as follows:

$$
\begin{aligned}
\sigma_{\mathrm{LL}}^{(r)}= & \int_{z_{\min }}^{1} d z_{1} \int_{z_{\min }}^{1} d z_{2} \int_{0}^{1} d z_{3} \int_{0}^{1} d z_{3} \int_{0}^{1} d \xi^{*} \int_{0}^{2 \pi} d \phi D^{(r)}\left(\frac{\gamma}{2}, z_{1}\right) D^{(r)}\left(\frac{\gamma}{2}, z_{2}\right) \\
& D^{(r)}\left(\frac{\gamma}{2}, z_{3}\right) D^{(r)}\left(\frac{\gamma}{2}, z_{4}\right) \frac{d \sigma^{\text {Born }}}{d \phi d \xi^{*}}\left(\phi, \xi^{*}\right) \Theta_{\xi_{a}}^{\xi_{b}}\left(\xi_{1}\right) \Theta_{\xi_{a}}^{\xi_{b}}\left(\xi_{2}\right),
\end{aligned}
$$

where $\phi$ is the azimuthal angle around the beam, $z_{1}, z_{2}$ denote fractions of the energies carried by the beams after emission of the initial-state photons, $z_{3}, z_{4}$ denote fractions of the energies carried by $e^{ \pm}$, in the process of the emission of the final-state photons, and the Born cross-section with pure $t$-channel photon exchange is

$$
\frac{d \sigma^{\text {Born }}}{d \phi d \xi^{*}}(\xi, \phi) \equiv \frac{2 \alpha^{2}}{s z_{1} z_{2}} \frac{1+\left(1-\xi^{*}\right)^{2}}{2\left(\xi^{*}\right)^{2}}
$$

where $\vartheta^{*}$ in $\xi^{*}=\left(1-\cos \vartheta^{*}\right) / 2=|t| /\left(s z_{1} z_{2}\right)$ is defined as the scattering angle in the LL hard-scattering rest frame. The built-in event selection is defined by $\Theta_{\xi_{a}}^{\xi_{b}}(\xi)=\Theta(\xi-$ $\left.\xi_{a}\right) \Theta\left(\xi_{b}-\xi\right)$ in terms of $\xi=(1-\cos \vartheta) / 2$ defined in the laboratory system. All notation is essentially the same as in Ref. [4]. The non-singlet LL structure functions $D^{(r)}(z, \gamma)$ up to $r=3$, with exponentiation, are from Ref. [11] and are also given explicitly in Ref. [4]. The Monte Carlo algorithm of the new LUMLOG is now accordingly extended to the case with the final-state bremsstrahlung, see Ref. $[13]^{5}$.

Now, we show explicitly the differential $\mathcal{O}\left(\gamma^{2}\right)_{B H L}$ cross section corresponding to BHLUMI with the matrix element of $\mathcal{O}\left(\alpha^{2}\right)_{\text {exp }}$ integrated analytically over the transverse photon momenta, keeping terms up to $\mathcal{O}\left(\gamma^{3}\right)$ :

$$
\sigma_{\mathrm{LL}}^{\mathrm{BHL}}=\int_{z_{\min }}^{1} d z_{1} d z_{2} \int_{0}^{1} d z_{3} d z_{4} \int_{0}^{1} d \xi^{*} \int_{0}^{2 \pi} d \phi \frac{d \sigma^{\mathrm{Born}}}{d \phi d \xi^{*}}\left(\phi, \xi^{*}\right) \Theta_{\xi_{a}}^{\xi_{b}}\left(\xi_{1}\right) \Theta_{\xi_{a}}^{\xi_{b}}\left(\xi_{2}\right) d_{B H L}^{(2)}\left(z_{1}, z_{2}, z_{3}, z_{4}\right)
$$

\footnotetext{
${ }^{5}$ In older LUMLOG only the initial-state emission was implemented.
} 

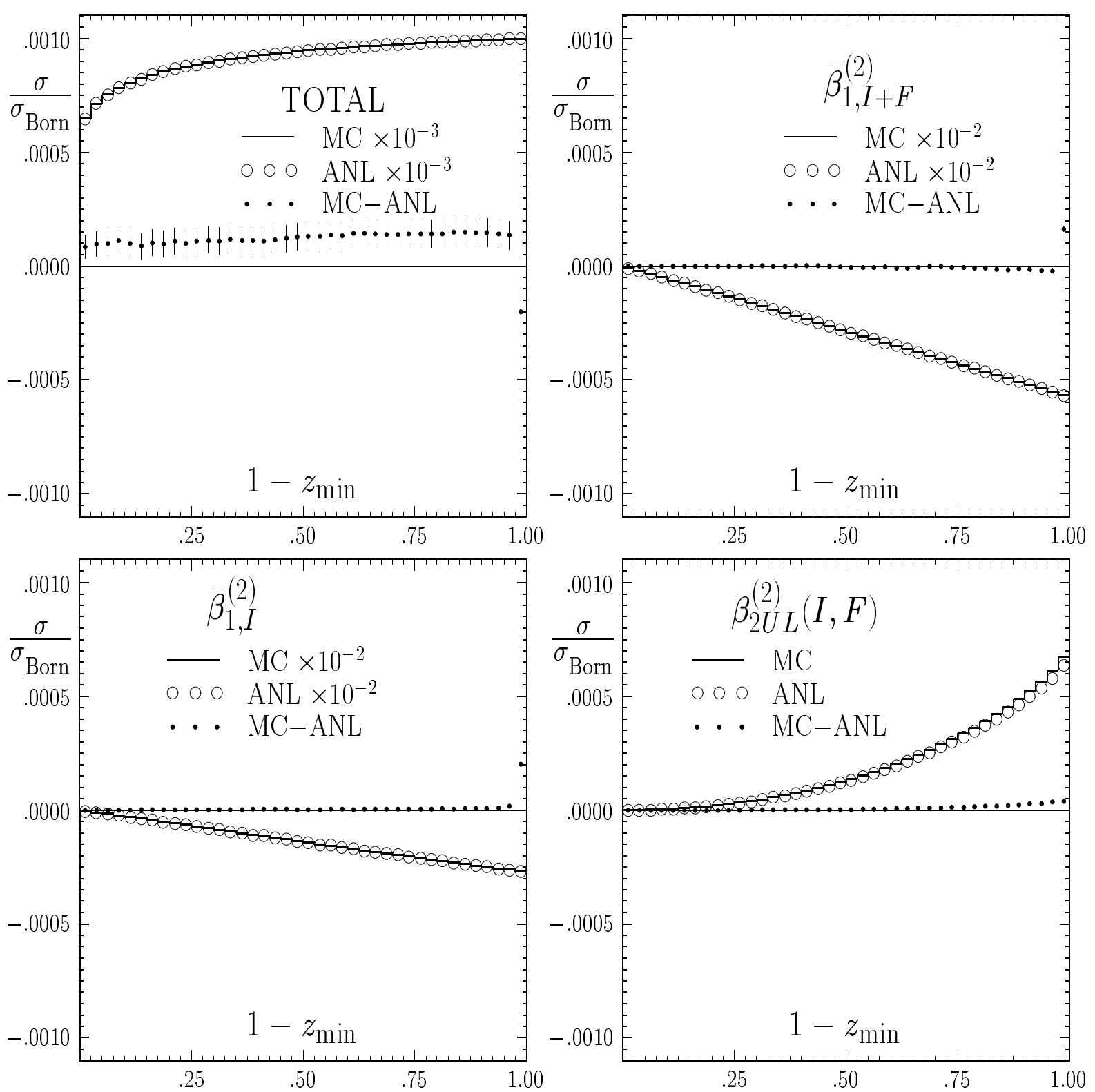

Figure 1: Comparison of LUMLOG (MC) and semi-analytical (ANL) results, see text for more explanations. 


$$
\begin{aligned}
& d_{B H L}^{(2)}\left(z_{1}, z_{2}, z_{3}, z_{4}\right)=z_{3} z_{4} f_{0}\left(\gamma / 2, v_{I}\right) f_{0}\left(\gamma / 2, v_{F}\right) f_{0}\left(\gamma / 2, v_{I}^{\prime}\right) f_{0}\left(\gamma / 2, v_{F}^{\prime}\right) e^{Y\left(\gamma, v_{F}\right)+Y\left(\gamma, v_{F}^{\prime}\right)} \\
& \quad\left\{B\left[\bar{\beta}_{0}\right](\gamma)+\sum_{K=I, F} \frac{B\left[\bar{\beta}_{1 K}^{(2)}\right]\left(\gamma, v_{K}\right)}{f_{0}\left(\gamma / 2, v_{K}\right)}+\sum_{L=I, F} \frac{B\left[\bar{\beta}_{1 L}^{(2)}\right]\left(\gamma, v_{L}^{\prime}\right)}{f_{0}\left(\gamma / 2, v_{L}^{\prime}\right)}\right. \\
& \quad+\sum_{K, L=I, F} \frac{B\left[\bar{\beta}_{2 U L}^{(2)}\right]\left(\gamma, K, L, v_{K}, v_{L}^{\prime}\right)}{f_{0}\left(\gamma / 2, v_{K}\right) f_{0}\left(\gamma / 2, v_{L}^{\prime}\right)} \\
& \left.\quad+\sum_{K, L=I, F} \frac{B\left[\bar{\beta}_{2 U U}^{(2)}\right]\left(\gamma, K, L, v_{K}, v_{L}\right)}{f_{0}\left(\gamma / 2, v_{K}\right) f_{0}\left(\gamma / 2, v_{L}\right)}+\sum_{K, L=I, F} \frac{B\left[\bar{\beta}_{2 L L}^{(2)}\right]\left(\gamma, K, L, v_{K}^{\prime}, v_{L}^{\prime}\right)}{f_{0}\left(\gamma / 2, v_{K}^{\prime}\right) f_{0}\left(\gamma / 2, v_{L}^{\prime}\right)}\right\}
\end{aligned}
$$

The following notation is used

$$
\begin{aligned}
& v_{I}=\left(1-z_{1}\right) z_{3}, \quad v_{F}=1-z_{3}, \quad v_{I}^{\prime}=\left(1-z_{2}\right) z_{4}, \quad v_{F}^{\prime}=1-z_{4}, \\
& Y(\gamma, v) \equiv-\gamma \ln (1-v)+\frac{1}{4} \gamma, \quad F(\gamma) \equiv \frac{\exp (-C \gamma)}{\Gamma(1+\gamma)}, \quad f_{0}(\gamma, v) \equiv F(\gamma) \gamma v^{\gamma-1} \\
& B\left[\bar{\beta}_{0}\right](\gamma)=1+\gamma+\frac{1}{2} \gamma^{2} \\
& B\left[\bar{\beta}_{1 K}^{(2)}\right](\gamma, v)=\bar{R}_{K}^{(2)}(\gamma, v) \\
& B\left[\bar{\beta}_{2 U U}^{(2)}\right]\left(\gamma, I, I, v_{I}, v_{F}\right)=f_{0}\left(\gamma / 2, v_{F}\right) \frac{1}{8} \gamma^{2} v_{I}^{\gamma / 2}\left(d_{0}^{*}\left(v_{I}\right)+\frac{1}{2} \gamma d_{1}^{*}\left(v_{I}\right)\right) \\
& B\left[\bar{\beta}_{2 U U}^{(2)}\right]\left(\gamma, F, F, v_{I}, v_{F}\right)=f_{0}\left(\gamma / 2, v_{I}\right) \frac{1}{8} \gamma^{2} v_{F}^{\gamma / 2}\left(d_{0}^{*}\left(v_{F}\right)+\frac{1}{2} \gamma d_{1}^{*}\left(v_{F}\right)\right) \\
& B\left[\bar{\beta}_{2 U U}^{(2)}\right]\left(\gamma, I, F, v_{I}, v_{F}\right)=\frac{1}{8} \gamma^{2} v_{I}^{\gamma / 2} v_{F}^{\gamma / 2}\left[k_{I F}^{*}\left(v_{I}, v_{F}\right)+\frac{1}{2} \gamma w_{I F}^{*}\left(v_{I}, v_{F}\right)\right] \\
& B\left[\bar{\beta}_{2 U L}^{(2)}\right]\left(\gamma, K, L, v_{K}, v_{L}^{\prime}\right)=\bar{R}_{K}^{(1)}\left(\gamma, v_{K}\right) \bar{R}_{L}^{(1)}\left(\gamma, v_{L}^{\prime}\right)
\end{aligned}
$$

where

$$
\begin{aligned}
& \bar{R}_{I}^{(2)}(\gamma, u)=F\left(\frac{1}{2} \gamma\right) u^{\frac{1}{2} \gamma}\{\gamma(-1 / 2+(1 / 4) u) \\
& \quad+\gamma^{2}(-1 / 2+(1 / 8) u)+\gamma^{2} \ln (1-u)(1 / 8-(1 / 16) u-1 /(8 u)) \\
& +\gamma^{3}(-(3 / 32) u)+\gamma^{3} \ln (1-u)(-1 / 32+(1 / 32) u) \\
& \left.\quad+\gamma^{3} \ln (1-u)^{2}(1 / 32-(1 / 64) u-1 /(32 u))+\gamma^{3} L i_{2}(u)(1 / 16-(1 / 32) u)\right\} \\
& \bar{R}_{F}^{(2)}(\gamma, u)=F\left(\frac{1}{2} \gamma\right) u^{\frac{1}{2} \gamma}\{\gamma(-1 / 2+(1 / 4) u) \\
& \quad+\gamma^{2}(-1 / 2+(1 / 8) u)+\gamma^{2} \ln (1-u)(-1 / 8+(1 / 16) u+1 /(8 u)) \\
& \quad+\gamma^{3}(-(1 / 32) u)+\gamma^{3} \ln (1-u)(+1 / 32-(1 / 32) u) \\
& \left.\quad+\gamma^{3} \ln (1-u)^{2}(-1 / 32+(1 / 64) u+1 /(32 u))+\gamma^{3} L i_{2}(u)(-1 / 16+(1 / 32) u)\right\} \\
& \bar{R}_{I}^{(1)}(\gamma, u)=\bar{R}_{F}^{(1)}(\gamma, u)=F\left(\frac{1}{2} \gamma\right) u^{\frac{1}{2} \gamma}\left\{\gamma(-1 / 2+(1 / 4) u)+\gamma^{2}(-(1 / 8) u)+\gamma^{3}(1 / 16) u\right\}
\end{aligned}
$$


and

$$
\begin{aligned}
w_{I F}^{*}\left(v_{1}, v_{2}\right) & =-\frac{1}{2} \frac{v_{1}}{1-v_{2}}+\ln \left(1-v_{1}\right)\left[-\frac{1}{2} \frac{1}{1-v_{2}}+\frac{1}{4} \frac{v_{1}}{1-v_{2}}+\frac{1}{4} \frac{v_{1}}{\left(1-v_{2}\right)^{2}}\right] \\
k_{I F}^{*}\left(v_{1}, v_{2}\right) & =k_{F I}^{*}\left(v_{2}, v_{1}\right)=\frac{1}{2}-\frac{1}{2} \frac{1}{1-v_{2}}+\frac{1}{4} \frac{v_{1}}{1-v_{2}}+\frac{1}{4} \frac{v_{1}}{\left(1-v_{2}\right)^{2}} \\
d_{0}^{*}(v) & =\frac{1}{2} v+\frac{1}{2}\left(1-\frac{v}{2}\right) \ln (1-v) \\
d_{1}^{*}(v) & =-\frac{3}{4} v-\frac{1}{4}(1-v) \ln (1-v)+\left(-\frac{1}{2}+\frac{1}{4} v\right) L i_{2}\left(\frac{-v}{1+v}\right)
\end{aligned}
$$

The $B\left[\bar{\beta}_{2 L L}^{(1)}\right]$ is functionally identical to $B\left[\bar{\beta}_{2 U U}^{(1)}\right]$.

In the following we shall discuss the content of Eq. (4) and show certain proofs we have that this formula is correct and correctly implemented in LUMLOG. Let us first observe that the differential cross section in Eq. (4) does not factorize into a product of the four functions dependent on $z_{i}=1,2,3,4$, as is the case for Eq. (1). It is generally expected because the integrand in Eq. (4) misses some $\mathcal{O}\left(\gamma^{3}\right)$. The version of Eq. (4) truncated to $\mathcal{O}\left(\gamma^{2}\right)$ does factorize, of course. In fact what we did was to check analytically that, in the difference

$$
d_{B H L}^{(2)}\left(z_{1}, z_{2}, z_{3}, z_{4}\right)-\prod_{i=1}^{4} D^{(3)}\left(\gamma / 2, z_{i}\right)
$$

all terms up to $\mathcal{O}\left(\gamma^{2}\right)$ cancel and only terms of $\mathcal{O}\left(\gamma^{3}\right)$ remain. The above is also a very essential cross-check of the proper implementation of the $\mathcal{O}\left(\gamma^{2}\right)$ in the matrix element of BHLUMI 4. $\mathrm{x}^{6}$. Thw next check is the following: we integrate analytically the integral of Eq. (4) over all variables except $t$ and $z=1-V=z_{1} z_{2} z_{3} z_{4}$, obtaining

$$
\begin{aligned}
\frac{d \sigma}{d|t| d z} & =\frac{4 \pi \alpha^{2}}{t^{2}} \frac{\left(1+(1-\xi)^{2}\right)}{2} F(2 \gamma) e^{2 Y(\gamma)} V^{2 \gamma}\left\{\frac{2 \gamma}{V}\left[1+\gamma+\frac{1}{2} \gamma^{2}\right]\right. \\
& +\gamma(-2+V)+\gamma^{2}(-2)+\gamma^{2} \ln (1-V)\left(3-3 V / 2-2 V^{-1}\right) \\
& +\gamma^{3}(-9 V / 8)+\gamma^{3} \ln (1-V)\left[2+1 / 8 V-2 V^{-1}-(1 / 4)(2-V)^{-} 1\right] \\
& +\gamma^{3} \ln (1-V)^{2}\left[-7 / 8+7 V / 16+(1 / 2) V^{-1}\right]+\gamma^{3} L i_{2}(V)(2-V) \\
& +\gamma^{3} \ln (1-V) \ln (2-V)(-1 / 4+V / 8)+\gamma^{3} L i_{2}((1-V) /(2-V))(1 / 4-V / 8) \\
& \left.+\gamma^{3} \operatorname{Li}_{2}(1 /(2-V))(-1 / 4+V / 8)-\gamma \xi \chi(V) /(1-V)\right\}
\end{aligned}
$$

We integrate the above using conventional non-MC methods:

$$
\sigma^{B H L}=\int_{|t|_{\min }}^{|t|_{\max }} d|t| \int_{z_{\min }}^{1} d z \frac{d \sigma}{d|t| d z}
$$

\footnotetext{
${ }^{6}$ The versions 4.02 and earlier of BHLUMI had some $\mathcal{O}\left(\gamma^{2}\right)$ bugs in the matrix element, which were found with the help of this cross-check.
} 
and we compare the result of this semi-analytical calculation with the MC result of LUMLOG. The result of the numerical comparison is shown in Fig. 1a as a function of $z_{\min }$. We get an agreement to within $1 \times 10^{-4}$. This is in fact the LL version of the analogous cross-check for multi-photon BHLUMI presented in Ref. [6]. We have done this test not only for the entire cross section, but also separately for the components coming from each $\bar{\beta}$ in Eq. (4). In Figs. 1b-1d we show the corresponding comparisons for the sum $\bar{\beta}_{1 I}^{(2)}+\bar{\beta}_{1 F}^{(2)}$, for $\bar{\beta}_{1 I}^{(2)}$ alone, and for $\bar{\beta}_{2 U L}^{(2)}$. If we have limited ourselves to the total cross section then, as known from Ref. [6], this test is not sensitive to the errors that cancel between initial and final state. Checking the components, for example from $\bar{\beta}_{1 I}^{(2)}$, which discriminate between initial and final state, strengthens our test considerably. In all cases we get good agreement below $1 \times 10^{-4}$. We believe that numerical implementation of the distributions of Eq. (4) in LUMLOG is correct at this precision level. Of course, what these tests do not check is whether Eq. (4) really represents BHLUMI to within $\mathcal{O}\left(\gamma^{3}\right)$. We shall come back to this question later. The other point is whether these tests extend to the event selection defined in terms of angles and not in terms of $|t|$. This point was discussed in Ref. [7], where the relevant additional tests were presented.

Having correctly implemented Eq. (4) in LUMLOG, we are now ready to answer the question: how big is the missing $\mathcal{O}\left(\gamma^{3}\right)$ in BHLUMI 4.x? The difference between the cross sections of Eq. (1) and Eq. (4) represents the missing $\mathcal{O}\left(\gamma^{3}\right)$ in BHLUMI. Since LUMLOG is an event generator, we can estimate the size of the missing $\mathcal{O}\left(\gamma^{3}\right)$ for arbitrary ES, including the real ones of the LEP experiments. In Fig. 2 we show four examples of the numerical results for the missing $\mathcal{O}\left(\gamma^{3}\right)$ for various ES's. All ES's except BARE1 are calorimetric, as the experimental ones. We keep non-calorimetric BARE1 because it is of some theoretical interest and because some $\mathcal{O}\left(\alpha^{2}\right)$ calculations [15] exist only for this ES. The most realistic ES is SICAL, which was used and is defined in Ref. [6]. The others were defined in the Bhabha Working Group of LEP2 Workshop and are described in detail in Ref. [2]. In particular the definition of the variable $z_{\min }$ in Fig. 2 depends on the type of ES. For BARE1 $z^{\text {BARE1 }}=s^{\prime} / s$, where $s^{\prime}$ is the effective mass squared of the final $e^{ \pm}$. In the LL approximation $z^{\mathrm{BARE} 1}=z_{1} z_{2} z_{3} z_{4}$, while for other ES's the exact definition of $z$ is rather complicated, see Ref. [6], let us only say that in LL approximation for these calorimetric ES's we have simply $z=z_{1} z_{2}$.

As we see from the results in Fig. 2, the missing $\mathcal{O}\left(\gamma^{3}\right)$ in BHLUMI is below $1 \times 10^{-4}$. This is consistent with the original estimates of Ref. [7] repeated in later papers ${ }^{7}$.

How important for the smallness of the missing $\mathcal{O}\left(\gamma^{3}\right)$ in the above test is the fact that we exponentiate? In other words, if we had at our disposal pure $\mathcal{O}\left(\alpha^{2}\right)$ calculation without exponentiation then would the missing $\mathcal{O}\left(\gamma^{3}\right)$ also be below $1 \times 10^{-4}$ or not? We find the answer to this question in Fig. 3, where we calculate the missing $\mathcal{O}\left(\gamma^{3}\right)$ in the case of a calculation without exponentiation. The results come from new LUMLOG, where we take the difference between $\mathcal{O}\left(\gamma^{3}\right)$ and $\mathcal{O}\left(\gamma^{2}\right)$ without exponentiation. As we see, for non-calorimetric BARE1 the missing $\mathcal{O}\left(\gamma^{3}\right)$ is as big as $15 \times 10^{-4}$, while for the realistic

\footnotetext{
${ }^{7}$ In Refs. $[2,3]$ the result of LUMLOG $2 \times 10^{-4}$ was quoted. This result was obtained from an early version of LUMLOG 4.3, which still had some bugs in the implementation of the contribution from $\bar{\beta}_{2}$.
} 

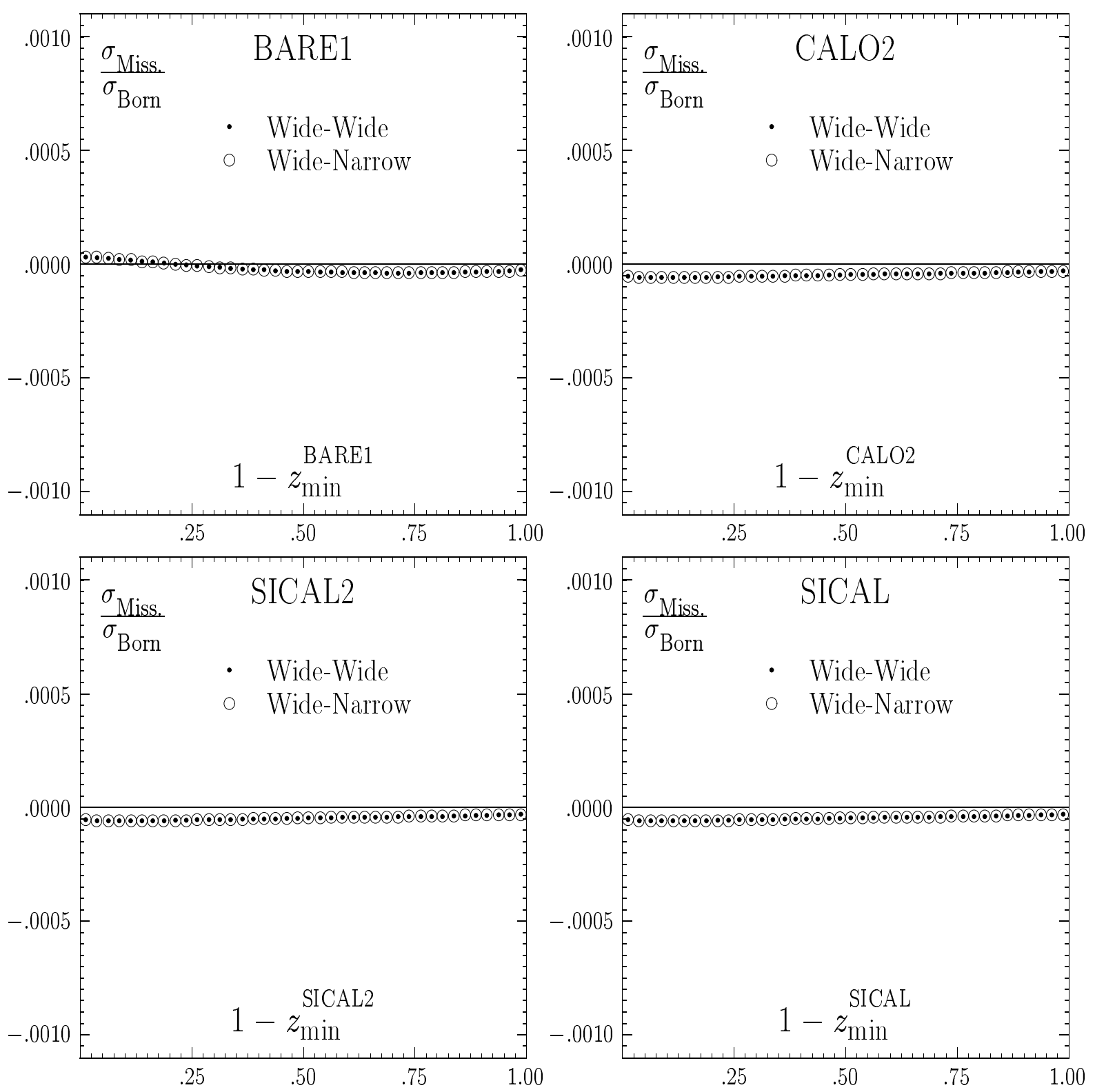

Figure 2: Missing photonic leading- $\log \mathcal{O}\left(\alpha^{3}\right)$ contribution $\sigma_{M i s s .}$ in the second-order BHLUMI 4.x without exponentiation calculated for various types of event selections using the LUMLOG 4.x.

calorimetric SICAL it reaches up to $3 \times 10^{-4}$. We therefore conclude that the $\mathcal{O}\left(\alpha^{2}\right)$ calculation, even the exact one, is insufficient for the present experimental precision of $8 \times 10^{-4}$ and especially for the ultimate LEP1 experimental precision of $5 \times 10^{-4}$. On the other hand, for the exponentiated $\mathcal{O}\left(\alpha^{2}\right)$ calculation we do need to worry about missing $\mathcal{O}\left(\alpha^{3}\right)$ corrections.

What is the main remaining uncertainty in the estimate of the missing $\mathcal{O}\left(\gamma^{3}\right)$ in this paper? In our opinion we still need at least one cross-check (one for calorimetric and 

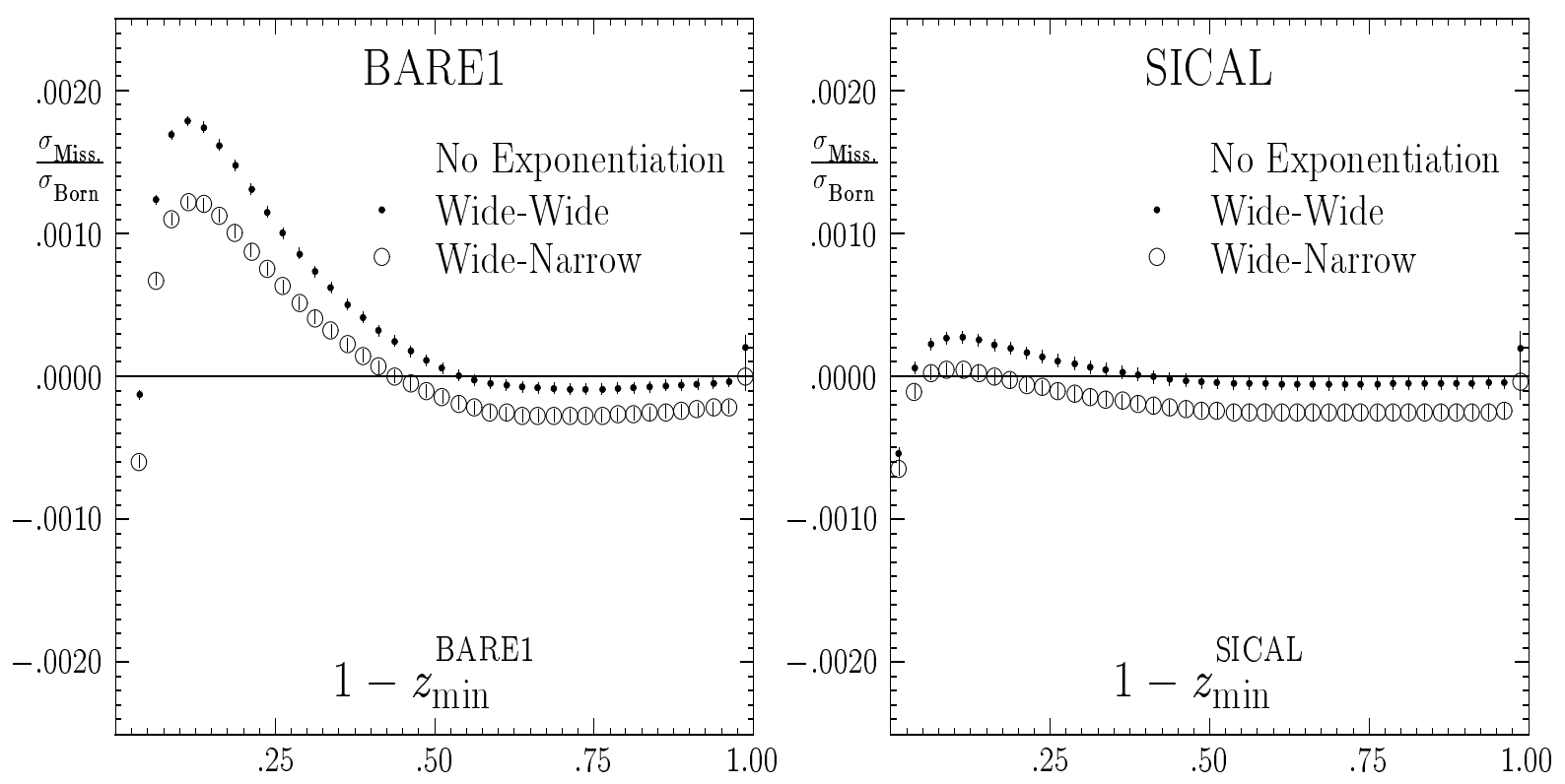

Figure 3: Missing photonic leading-log $\mathcal{O}\left(\alpha^{3}\right)$ contribution $\sigma_{\text {Miss. }}$ in the multi-photon MCEG BHLUMI 4.x calculated for various types of event selections using the MCEG LUMLOG 4.x.

one non-calorimetric ES), which shows that analytical integration of BHLUMI matrix element leading to Eq. (4) is correct. In Ref. [6] we present the analytical results as in Eq. (4), but also with terms $\mathcal{O}(\alpha \gamma)$; this agrees perfectly with the results of BHLUMI MC. This was done for a non-calorimetric ES and we are confident that both BHLUMI and its analytical integration are correct for the non-calorimetric class of ES's. We have to repeat this kind of cross-check for the (more difficult) case of at least one example of calorimetric ES. Such an exercise will be completed soon. In the mean time we apply in the present paper a "safety factor" of 2 in our estimate of the missing $\mathcal{O}\left(\gamma^{3}\right)$ so our resulting main conclusions are the following: The missing third-order leading-logarithmic correction to small-angle Bhabha second-order exponentiated calculation, as implemented in the BHLUMI 4.04 event generator is negligible, below $2 \times 10^{-4}$, for the typical LEP luminometric event selection. This conclusion gets substantially weakened (a factor of 3 worse) for the calculation without exponentiation. The above result together with recent results on the second-order sub-leading corrections, see refs. [15-18], creates new framework in which we hope to be able to reduce total theoretical error in the small-angle Bhabha calculation below the elevl of $0.1 \%$.

\section{Acknowledgements}

Authors would like to thank the CERN Theory Division for hospitality and partial support during completion of this work. They are also very grateful for the support of the ALEPH and OPAL Collaborations. 


\section{References}

[1] LEP Electroweak Working Group, Technical report, CERN LEPEWWG/95-02, (unpublished), A Combination of Preliminary LEP Electroweak Results from the 1995 Summer Conferences, see also ALEPH 95-093 PHYSIC 95-086, DELPH 95-137 PHYS 562, L3 Note 1814, OPAL Technical Note TN312.

[2] S. Jadach and O. Nicrosini, (convenors of Bhabha Working Group), in Physics at LEP2, CERN Yellow Report 96-01, edited by G. Altarelli, T. Sjöstrand, and F. Zwirner (CERN, Geneva, 1996), Vol. 2, p. 229, hep-ph/9602393.

[3] S. Jadach and O. Nicrosini et al., Phys. Lett. B, (1996), in print, hep-ph/9605239.

[4] S. Jadach, E. Richter-Wạs, B. F. L. Ward, and Z. Wa̧s, Phys. Lett. B268, 253 (1991).

[5] S. Jadach et al., in Tennessee International Symposium on Radiative Corrections: Status and Outlook, edited by B. F. L. Ward (World Scientific, Singapore, 1995), Gatlinburg, Tennessee, USA, June 1994, see also CERN preprint CERN-TH.7452/94.

[6] S. Jadach, E. Richter-Wąs, B. F. L. Ward, and Z. Wąs, Phys. Lett. B353, 362 (1995), CERN preprint CERN-TH/95-38.

[7] S. Jadach, E. Richter-Wąs, B. F. L. Ward, and Z. Wa̧s, Phys. Lett. B260, 438 (1991).

[8] F. A. Berends and R. Kleiss, Nucl. Phys. B228, 537 (1983).

[9] F. A. Berends, R. Kleiss, and W. Hollik, Nucl. Phys. B304, 712 (1988).

[10] S. Jadach, B. F. L. Ward, and M. Skrzypek, Phys. Lett. B257, 173 (1991).

[11] M. Skrzypek, Acta Phys. Pol. B23, 135 (1992).

[12] S. Jadach, E. Richter-Wạs, B. F. L. Ward, and Z. Wạs, Comput. Phys. Commun. 70, 305 (1992).

[13] S. Jadach et al., preprint CERN TH/96-158, June 1996, "Upgrade of the Monte Carlo program BHLUMI for Bhabha scattering at low angles to version 4.04", to be submitted to Comput. Phys. Commun.; available from the WWW location http://hpjmiady.ifj.edu.pl (unpublished).

[14] S. Jadach and B. F. L. Ward, Semi-Analytical Third Order Calculations of the Small Angle Bhabha Cross Sections, 1996, to be published in Acta Phys. Pol.

[15] A. B. Arbuzov, E. A. Kuraev, N. P. Merenkov, and L. Trentadue, preprint CERNTH/95-241 (unpublished).

[16] S. Jadach, M. Melles, B. F. L. Ward, and S. A. Yost, Phys. Lett. B377, 168 (1996), hep-ph/9603248. 
[17] G. Montagna, O. Nicrosini, and F. Piccinini, Phys. Lett. B, (1996), in print; also preprint FNT/T-96/8, hep-ph/9605252.

[18] S. Jadach, M. Melles, B. F. L. Ward, and S. Yost, in $Q C D$ and $Q E D$ in Higher Order: 1996 Zeuthen workshop on elementary particles, edited by J. Bluemlein (Elsevier Science Publishers, Amsterdam, 1996), Rheinsberg, Germany, April 21-26, 1996. 